\title{
Functional and immunogenic characterization of diverse HCV glycoprotein E2 variants
}

Tanvi Khera ${ }^{1 \#}$, Patrick Behrendt ${ }^{1,2,11 \#}$, Dorothea Bankwitz ${ }^{1}$, Richard J.P. Brown ${ }^{1}$, Daniel Todt ${ }^{1,12}$, Mandy Doepke ${ }^{1}$, Abdul Ghafoor Khan ${ }^{3}$, Kai Schulze ${ }^{4}$, John Law ${ }^{5}$, Michael Logan ${ }^{5}$, Darren Hockman ${ }^{5}$, Jason Alexander Ji-Xhin Wong, Leona Dold ${ }^{6,7}$, Victor Gonzalez-Motos ${ }^{8}$, Ulrich Spengler ${ }^{9}$, Abel ViejoBorbolla $^{8}$, Luisa Ströh ${ }^{8}$, Thomas Krey ${ }^{8,11}$, Alexander W. Tarr ${ }^{10}$, Eike Steinmann ${ }^{1,11,12}$, Michael P. Manns $^{2,11}$, Florian Klein ${ }^{6,7}$, Carlos A. Guzman ${ }^{4}$, Joseph Marcotrigiano ${ }^{3}$, Michael Houghton ${ }^{5}$, Thomas Pietschmann $^{1,11^{*}}$

${ }^{1}$ Institute of Experimental Virology, TWINCORE, Centre for Experimental and Clinical Infection Research; a joint venture between the Medical School Hannover (MHH) and the Helmholtz Centre for Infection Research (HZI), Hannover, Germany

${ }^{2}$ Department of Gastroenterology, Hepatology and Endocrinology, Hannover Medical School, Hannover, Germany

${ }^{3}$ National Institute of Allergy and Infectious Diseases, National Institutes of Health, Bethesda, MD 208929806, USA

${ }^{4}$ Department of Vaccinology and Applied Microbiology, Helmholtz Centre for Infection Research, Braunschweig, Germany

${ }^{5} \mathrm{Li}$ Ka Shing Institute of Virology, Department of Medical Microbiology \& Immunology, University of Alberta, Edmonton, Canada

${ }^{6}$ Institute of Virology, Center for Molecular Medicine Cologne (CMMC), University of Cologne, Cologne, Germany.

${ }^{7}$ German Centre for Infection Research (DZIF), partner site Cologne, Germany,

${ }^{8}$ Institute of Virology, Hannover Medical School, 30625 Hannover, Germany

${ }^{9}$ Department of Internal Medicine 1, Rheinische Friedrich-Wilhelms-University Bonn, Bonn, Germany

${ }^{10}$ NIHR Nottingham Digestive Diseases Biomedical Research Centre and School of Life Sciences, The University of Nottingham, Nottingham, UK

${ }^{11}$ German Centre for Infection Research (DZIF), partner site Hannover-Braunschweig, Germany

${ }^{12}$ Department of Molecular and Medical Virology, Ruhr-University Bochum, Bochum, Germany

\# equally contributing authors

*Correspondence Address: Prof. Dr. rer. nat. Thomas Pietschmann, Institute of Experimental Virology, Twincore, Centre for Experimental and Clinical Infection Research, Feodor-Lynen Str 7-9, 30625 Hannover, thomas.pietschmann@twincore.de, Phone: +49-511-220027130, FAX +49-511-220027139.

Keywords: HCV, Glycoproteins, immunogen, antibodies, recombinant proteins Word count: 5678

Conflict of interest: The authors declare no conflicts of interest that pertain to this work. 


\section{Abstract}

36 Background and Aims Induction of cross-reactive antibodies targeting conserved epitopes of the 37 envelope proteins E1E2 is a key requirement for an HCV vaccine. Conserved epitopes like the viral 38 CD81-binding site are targeted by rare broadly neutralizing antibodies. However, these viral segments are 39 occluded by variable regions and glycans. We aimed to identify antigens exposing conserved epitopes and 40 to characterize their immunogenicity.

41 Methods We created HCV variants with mutated glycosylation sites and/or hypervariable region 1 42 (HVR1). Exposure of the CD81 binding site and conserved epitopes was quantified by soluble CD81 and 43 antibody interaction and neutralization assays. E2 or E1-E2 heterodimers with mutations causing epitope 44 exposure were used to immunize mice. Vaccine-induced antibodies were examined and compared with 45 patient-derived antibodies.

46 Results Mutant viruses bound soluble CD81 and antibodies targeting the CD81 binding site with 47 enhanced efficacy. Mice immunized with E2 or E1E2 heterodimers incorporating these modifications 48 mounted strong, cross-binding, and non-interfering antibodies. E2-induced antibodies neutralized the 49 autologous virus but they were not cross-neutralizing.

50 Conclusions Viruses lacking the HVR1 and selected glycosylation sites expose the CD81 binding site and 51 cross-neutralization antibody epitopes. Recombinant E2 proteins carrying these modifications induce 52 strong cross-binding but not cross-neutralizing antibodies.

\section{Lay summary}

54 Conserved viral epitopes can be made considerably more accessible for binding of potently neutralizing 55 antibodies by deletion of HVR1 and selected glycosylation sites. Recombinant E2 proteins carrying these 56 mutations are unable to elicit cross-neutralizing antibodies suggesting that exposure of conserved epitopes 57 is not sufficient to focus antibody responses on production of cross-neutralizing antibodies.

\section{$58 \quad$ Highlights}

59 - High resolution mapping of the impact of HVR1 and glycosylation sites on CD81 binding, 60 antibody binding and virus neutralization

61 - Viral mutants lacking HVR1 and selected glycosylation sites are functional and they expose the 62 viral CD81 binding site and conserved cross-neutralization epitopes

63 - E2 proteins with these mutations induce cross-binding and non-interfering antibodies in mice 


\section{Introduction}

65 Hepatitis $\mathrm{C}$ virus (HCV) is a global health burden affecting approximately 71 million people worldwide 66 [1]. Infection often leads to chronic hepatitis, with the subsequent risk for liver cirrhosis and 67 hepatocellular carcinoma. Persistent HCV infection is now curable with the introduction of direct-acting 68 antivirals (DAAs). However, a prophylactic HCV vaccine is not available. Since viral re-infection is 69 possible and as many HCV infected individuals are not diagnosed, a vaccine against HCV would facilitate 70 global HCV eradication programs.

71 The extreme diversity of HCV is a major obstacle for vaccine development [2]. The HCV E1E2 proteins 72 are essential for viral cell entry, they bind the HCV receptor CD81 and they are targets for neutralizing 73 antibodies. Hence, immunogens based on E1E2 represent one major branch of vaccine development [3, 4] 74 and numerous approaches to induce E1E2-targeting broadly neutralizing antibodies (bNabs) have been 75 explored [5]. For recombinant E1E2, the most advanced HCV subunit vaccine candidate, induction of 76 robust cross-binding and cross-neutralizing antibody responses was observed in multiple animal models 77 and in humans $[4,6,7,8]$.

$78 \mathrm{HCV}$ has evolved mechanisms to evade humoral immune responses including high functional flexibility 79 and variability of immunogenic portions of its envelope proteins [9]. The highest sequence variability occurs in the first 27 amino acids of the $\mathrm{N}$-terminus of $\mathrm{E} 2$, which is referred to as the hypervariable region 81 (HVR1), and which is dispensable for HCV infection in vitro [10, 11]. The HVR1 is immunogenic and 82 most patients mount antibodies targeting this region [12]. However, these antibodies rapidly select 83 resistant viral variants [13]. Deletion of the HVR1 renders HCV more susceptible to antibody 84 neutralization, and it increases virus binding to soluble CD81, suggesting that this region occludes key 85 neutralization epitopes and the viral CD81 binding site [10, 11]. HCV E1E2 heterodimers are also heavily 86 glycosylated at multiple sites both within E1 and E2 and glycans modulate glycoprotein function and 87 antibody neutralization $[14,15,16]$. Structural analyses of the E2 core domain show that the conserved 88 CD81 binding site is surrounded by several glycosylation sites and that it overlaps with the epitopes of 89 bNabs isolated from patients like for instance $\mathrm{HC} 1$ and $\mathrm{HC} 11$ [17, 18, 19, 20] (Fig. 1). Strikingly, in vitro $90 \mathrm{HCV}$ is unable to escape antibody pressure by $\mathrm{HC}-1$ and $\mathrm{HC}-11$ suggesting that immune responses 91 targeting these epitopes may confer robust protection [21, 22].

92 We hypothesized that the HVR1 and protein glycosylation limits access to these conserved viral epitopes. 93 In turn, immunogenicity of these epitopes may be low and antibodies targeting these viral regions may 94 arise only very infrequently. To overcome this limitation and to focus immune responses to conserved 
viral epitopes we combined targeted protein deglycosylation with deletion of HVR1 to create viruses and proteins with increased exposure of the crucial CD81 binding site and possibly superior immunogenicity.

\section{Results}

\section{Inactivation of glycosylation sites and deletion of HVR1 increase exposure of conserved neutralization epitopes and the CD81 binding site}

To generate an improved HCV vaccine antigen, we aimed to increase the exposure of the CD81-binding site by combined mutation of the HVR1 and selected glycosylation sites. Jc1 reporter viruses expressing the J6 (GT2a) E1E2 proteins [23, 24] were constructed harboring $\mathrm{N}$ to A substitutions at glycosylation sites N417 (417) (numbering according to the H77 reference stain; J6 numbering in brackets), N423 (423), N448 (448), N532 (534) and N645 (649) (Fig. 1A). A previous report had shown that mutations at these residues still allowed viral entry of JFH1 and can therefore be studied and further evaluated in vitro [15]. Molecular modelling of the glycans associated with the HCV E2 core structure revealed that some were proximal to the CD81 binding site and conserved neutralization epitopes (Fig. 1B). Therefore, to further increase exposure of the CD81 binding site, we combined these mutations with the deletion of the HVR1. Fitness of mutant viruses was examined by transfection of Huh7-Lunet N\#3 cells that do not express CD81 [25], and therefore do not permit reinfection of the cells. Replication of all viral mutants was comparable to wild type (WT) Jc1 (Fig. 1C). Huh-7.5 cells were inoculated with virus stocks after normalization to viral core protein that acts as a marker for virus particle release. In line with our previous report [10], deletion of HVR1 resulted in a 5-fold reduction of specific infectivity of Jc1 (Fig. 1D). Likewise, point mutations of individual glycosylation sites were well tolerated as specific infectivity was unaffected or at most reduced by ca. 5-fold.

To examine the impact of these mutations on the exposure of conserved neutralization epitopes within the CD81 binding site, we used the soluble, large-extracellular loop of CD81 (CD81-LEL) and two potent bNabs (HC-1 and HC-11) that target the CD81 binding site [17, 18, 19, 20, 21], for precipitation of the indicated viral mutants (Fig. 2A and B). Deglycosylation of specific residues in the parental Jc1 virus slightly increased binding of $\mathrm{HC}-1$ in particular for mutant N448A and N534A as is evidenced by enhanced core protein precipitation compared to Jc1, without being statistical significant (Fig. 2A). However, the deletion of HVR1 significantly enhanced precipitation by this antibody, which did not further increase by additional point mutations of glycosylation sites at indicated positions (Fig. 2A). Similar results were observed when the HC-11 antibody was used: mutation of individual glycosylation sites increased antibody binding (e.g. N423A, N448A). Deletion of HVR1 had the greatest effect, which was not further boosted by mutation of the glycosylation sites (Fig. 2B). Comparable results were obtained 4 
when we used CD81-LEL for precipitation. In the context of parental Jc1, inactivation of individual glycosylation sites modestly increased precipitation (Fig. 2C). As may be expected from the available structural information (Fig. 1B) [17, 18], the effect was greatest for mutants N423A and N534A, as these glycosylation sites are directly adjacent to the CD81 binding site. Deletion of HVR1 had the greatest effect and precipitation of viruses lacking HVR1 was not further boosted by deglycosylation of specific residues.

\section{Deletion of HVR1 and glycosylation sites enhances virus neutralization}

To characterize the accessibility of HCV antibody epitopes, we performed neutralization assays with a panel of bNAbs (Fig. 3A and $\mathrm{C}$ and supplementary table 1). Moreover, we quantified virus neutralization by CD81-LEL (Fig. 3B and C and supplementary table 1). Dose dependence of neutralization for selected viruses is displayed in figure 3C. Radar plots indicating the inhibitory concentration $90 \%$ ( $\mathrm{IC}_{90}$ ) of different antibodies or the CD81-LEL against parental HCV and all mutant viruses is displayed in figure 3A and 3B. Each plot shows a specific virus and its sensitivity towards given antibodies (Fig. 3A), whereas figure panel 3B highlights the susceptibility of each virus to competition by CD81-LEL. In the context of parental Jc1, deletion of glycans at position N423 and N448 increased the susceptibility to all tested antibodies, whereas removal of glycosylation at residues N417, N534, and N649 had little effect (Fig. 3A upper panels). As expected, deletion of HVR1 enhanced neutralization by all antibodies tested. Combination of deletion of HVR1 with deletion of specific N-glycosylation sites modified neutralization by these antibodies, and the Jc1- $\Delta$ HVR1-N534A mutant exhibited a further enhanced susceptibility to neutralization by HC-1 and HC-11 (Fig. 3A and 3C). Removal of glycosylation at the N423, N534, and N417 site in the context of WT Jc1 enhanced inhibition of infection by CD81-LEL, whereas mutation of the N417 and N649 sites had no effect (Fig. 3B). Deletion of HVR1 increased neutralization by CD81LEL and this was further enhanced ca. 5-10-fold by addition of the N534 mutation. Therefore, combined deletion of HVR1 and inactivation of glycosylation site N534 enhanced antibody and CD81 binding to virus particles and it had the most drastic effect on neutralization by bNabs and soluble CD81.

Next, we evaluated neutralization of Jc1, Jc1-N534A, Jc1- $\Delta$ HVR1 and Jc1- $\Delta$ HVR1-N534A by sera from individuals chronically infected with HCV GT 1 and 2 (Fig. 4A-C). Similar to the results of neutralization with monoclonal antibodies and CD81-LEL (Fig. 3), the sera poorly neutralized Jc1. Neutralization was enhanced by elimination of the N534 site in Jc1, further boosted by deletion of HVR1, and it was maximal when the deletion of HVR1 was combined with the inactivation of the N534 glycosylation site with minor interpatient variation, independent of the patients` HCV genotype (Fig. 4A-C). Thus, combined deletion of HVR1 and inactivation of glycosylation site N534 enhanced antibody and CD81 binding to virus particles and it had the most drastic effect on neutralization by bNabs and soluble CD81 and patient sera. As these 5 
viruses are infectious they present well-folded and functional viral envelope proteins that display conserved epitopes in a more accessible manner. Thus, envelope proteins carrying these modifications,

162

163

particularly the deletion of HVR1 combined with the N534A mutation, may constitute good candidates for focusing antibody responses on conserved viral epitopes.

\section{Immunogenicity of recombinant E2 proteins with and without HVR1 and glycosylation}

165 To test this, we prepared soluble, truncated HCV J6 E2 protein variants in 293T cells and immunized $166 \mathrm{BALB} / \mathrm{c}$ mice. These proteins have been previously reported to inhibit virus infection indicating that they 167 are properly folded [26]. Supplementary figure 1 shows the immunization scheme. Briefly, animals 168 received $30 \mu \mathrm{g}$ of soluble E2 injected together with bis-(3',5')-cyclic dimeric adenosine monophosphate 169 (c-di-AMP) as adjuvant and were boosted thrice with the same formulation. As control, animals were vaccinated with PBS and adjuvant only. We also used two vaccination series where we changed antigens in each boost in order to promote the stimulation of antibodies specific for the conserved epitopes. In sequence A animals were primed using WT J6 E2 (GT2a), followed by three boosters with E2 $\Delta$ HVR1 (J6 GT2a derivative), E2 $\triangle$ HVR1/N534A (J6 GT2a derivative), and WT Con1 E2 (GT1b), respectively. In sequence B mice were primed with E2 $\triangle \mathrm{HVR} 1 / \mathrm{N} 534 \mathrm{~A}$, followed by three booster immunizations with E2 $\Delta$ HVR1 (J6 GT2a derivative), WT Con1 (GT1b) E2, and WT J6 (GT2a) E2, respectively. Finally, to examine the relevance of global glycosylation for antigenicity, we vaccinated with WT J6 (GT2a) or with J6 (GT2a) E2 $\triangle$ HVR1 that had been deglycosylated by PNGaseF treatment prior to vaccination.

The binding of vaccine-induced antibodies to $293 \mathrm{~T}$ cell derived recombinant E2 proteins or E1-E2 heterodimers from HCV infected cells was determined by ELISA (Fig. 5 and supplementary figure S2). Fig 5A shows end point titers of sera from vaccinated animals reacting with H77 (GT1a), Con1 (GT1b), J6 (GT2a) or J6AHVR1 (GT2a) recombinant proteins. High binding titers across these GT1 and GT2 antigens were observed in all vaccinated groups up to a maximal end point dilution approaching 10E6 (Fig. 5A). Moreover, irrespectively of which protein was used for vaccination, most of these antibodies targeted binding sites outside of the HVR1, as end point titers were comparable between J6 (GT2a) and J6AHVR1 (GT2a) ELISA antigens. Cross-binding activity to these recombinant E2 proteins was clearly enhanced for vaccination with E2 $\Delta \mathrm{HVR}$ compared to wildtype recombinant E2 (Fig.5C left panel). Moreover, vaccination according to sequence A and B resulted in greater cross-binding to recombinant E2 proteins. To evaluate the binding of these antibodies to heterodimeric E1-E2 protein complexes from different HCV strains, we used plates coated with galanthus nivalis lectin (GNA) to capture HCV E1E2 complexes expressed in Huh-7.5 cells transfected with infectious HCVcc chimeras. Subsequently, these plates were incubated with sera from our vaccinated animals and end point binding titers were calculated 
(Fig. 5B). In each group of vaccinated animals, anti-E2 antibodies binding to all examined E1-E2 protein complexes were detectable. Thus, each vaccination approach induced broadly cross-binding antibodies that recognize E2 proteins of all major HCV GTs. With exception of the vaccination with J6 $\Delta \mathrm{HVR}-534$ E2 protein, however, all vaccination approaches induced antibodies with cross-binding activity to these E1-E2 heterodimers indistinguishable from the ones induced by parental J6 E2 (Fig. 5B and 5C right panel). Taken together, all vaccination approaches induced strong cross-binding antibodies to E2 and E1E2 heterodimers. Vaccination with different E2 proteins, most notably with E2 $\Delta H V R 1$, enhanced crossbinding when judged by ELISAs involving recombinant E2. However, this difference was not detected when cross-binding was quantified with ELISAs based on E1-E2 heterodimers from cells replicating infectious virus.

\section{Evaluation of neutralizing activity of vaccine induced antibodies}

To examine autologous virus and virus cross-neutralizing responses we incubated Jc1 and GT5a reporter viruses, respectively, with increasing doses of purified IgG from vaccinated animals or from patient sera (Fig. 6A). The autologous virus Jc1 was neutralized by antibodies from the animals vaccinated with deglycosylated E2, with deglycosylated $\triangle \mathrm{HVR} 1$ or the series A antigen combinations. The strength of these autologous neutralizing antibody responses was at least as strong as the neutralization by polyclonal human antibodies collected from a panel of GT1, 2 and 3 chronically infected individuals. This correlated with comparable binding of patient-derived and vaccine-induced antibodies to recombinant E2 and virus particle associated E2, quantified by surface plasmon resonance and virus particle precipitation (supplementary figure S4). However, none of the vaccine-induced antibodies were able to cross-neutralize the GT5a reporter virus which was at least partially neutralized by the majority of patient derived antibodies.

It has been reported that $\mathrm{HCV}$ induces antibodies that bind to E2 and that interfere with the activity of neutralizing antibodies, thereby masking vaccine-induced antibody neutralization [27, 28]. To explore whether our vaccine candidates induced such interfering antibodies, we used pooled polyclonal mouse IgGs from each vaccinated group and mixed them with polyclonal IgGs from a patient that displays strong neutralizing activity. The neutralization efficacy was not decreased by addition of IgG vaccinated mice, suggesting that the vaccines did not induce antibodies interfering with neutralization (Fig. 6B).

The role of glycans and the HVR1 for immunogenicity was also assessed using protein from a different genotype (GT1a H77), as well as in the context of E1E2 heterodimers purified from CHO cell extracts. A detailed description of the purification procedure is provided in the materials and methods section and the quality control of the purification is given in supplementary figure S5A. The binding of E1E2 (WT), E1E2 
4224 (N417Q) and E1E2 (N532Q) antigens to CD81-LEL was examined by ELISA and revealed enhanced 227 of E2 specific antibody responses comparable between all groups (Fig. 7B). As shown in figure 7C all 10 three vaccine candidates induced antibodies competing with binding of the known monoclonal antibodies AR3B, 1:7, HC33.4 and AP33 as assessed by a competition assay. Finally, we used GT1a H77 HCVpp assays to examine neutralization efficacy of vaccine induced antibodies. Each vaccine candidate induced 


\section{Discussion}

Here we provide a high resolution map of viral determinants that govern exposure of the CD81 binding site and of conserved neutralization epitopes. We show that these changes are well tolerated indicating that they do not abrogate the functioning of E1/E2. The most global change in antibody binding/neutralization and CD81 binding/neutralization was accomplished by deletion of HVR1 combined with inactivation of glycosylation site 534. Thus, we chose this combined modification for vaccination approaches involving recombinant proteins. To rule out strain-specific differences between immunogens, we created these variants in the background of J6 (GT2a) E2 and in the context of the H77 (GT1a) strain. We prepared H77 E1E2 heterodimers from CHO cell extracts and recombinant E2 protein secreted from 293 T cells to examine the importance of producer cells and the relevance of E1. To globally assess the relevance of glycosylation in our vaccine candidates we also examined the immunogenicity of 293T cellderived proteins that had been enzymatically deglycosylated prior to vaccination. Finally, we included a vaccination protocol involving variable immunogens and in each case at least one immunogen from an alternative viral GT.

All vaccination approaches induced robust cross-binding antibody responses as determined by ELISA assays involving recombinant proteins or cell extracts expressing E1-E2 heterodimers from all major HCV GTs (Fig. 5). Vaccination with E1-E2 heterodimers induced antibodies that competed with the binding of previously described bNAbs AR3B, 1:7, HC33.4 and AP33 and we detected virus neutralization in HCVpp assays (Fig. 7). However, inactivation of N417 or N532 glycosylation sites in the context of H77 E1E2 heterodimers did not grossly affect these responses although the mutation N532Q mediated increased binding of the recombinant E1E2 complex to CD81. Among vaccinations with 293T-derived E2 proteins immunization with J6-E2 $\triangle \mathrm{HVR} 1$ and the two immunization series A and B mounted superior cross-binding antibodies compared with J6-E2 and the other examined approaches. However, this was only detected when recombinant E2 proteins from 293T cells were used as ELISA antigens and not when E1-E2 heterodimers partially purified from HCV replicating cells were employed. Thus, some of our E2protein based vaccination approaches successfully improved production of cross-binding antibodies to the recombinant immunogens but not to the E1-E2 heterodimer expressed in infected cells. Thus, apparently there are structural differences between these proteins and E1-E2 heterodimers. These differences could impact on vaccine efficacy and they should be considered when standardizing tests between laboratories and when estimating cross-binding between different studies. It is also possible that such structural differences were responsible that vaccination with 293T-derived E2 proteins triggered only modest autologous neutralizing antibodies and no cross-neutralization. It has been shown that purified, UVinactivated HCVcc particles induce cross-neutralizing antibodies in mice and non-human primates [29, 
30]. Thus, it will be interesting to explore if vaccination with the HCVcc variants characterized here induces a focused and enhanced neutralizing antibody response.

It is surprising that vaccination with E2 from $293 \mathrm{~T}$ cells induced very robust, cross-binding antibodies that, however, did not cross-neutralize. One possible explanation was that these immunogens induced antibodies that interfere with cross-neutralization as has been described previously [27, 28]. However, the vaccine induced antibodies did not interfere with cross-neutralization of HCV patient derived polyclonal antibodies (Fig. 6). Thus, we believe that the 293T-derived E2 vaccines examined here efficiently induced antibodies that target conserved binding epitopes, but that targeting these epitopes is not crossneutralizing. It is possible that this reflects in part a species-specific limitation of immunogenicity of recombinant E2 proteins in mice as recent results by other groups and us suggest that anti-HCV neutralizing antibody responses are more readily induced in guinea pigs [31,32]. Although neutralizing antibodies were elicited, those were primarily strain-specific, thus explaining the neutralization of autologous Jc1 particles. Notably, the conserved CD81 binding site seems to be structurally highly flexible, and it was proposed that this flexibility may favor induction of non-neutralizing antibodies [33]. In line with this, it is possible that the recombinant E2 proteins used here preferentially adopt a conformation that induces binding, but non-neutralizing antibodies and that the conformation inducing neutralizing antibodies may be under represented in these recombinant proteins. Thus, epitope exposure alone may be insufficient and should be complemented by vaccine design aiming at rigidifying this region.

The lack of robust immunocompetent animal models permissive for HCV makes it hard to assess if the quantity and quality of vaccine-induced immunity is sufficient to confer protection. Thus, it is difficult to predict to which extent antibody responses induced by current vaccination approaches, including the ones presented here, contribute to protection. In vivo, antibody functions independent of direct virus particle neutralization could facilitate protection. For instance antibody dependent cytotoxicity (ADCC) may contribute to clearance of $\mathrm{HCV}$ infected cells. In this regard, binding, but non-neutralizing antibodies may contribute to HCV protection, as has been recently reported for an HIV infection in vivo [34]. Therefore, development of vaccination approaches inducing strong $\mathrm{T}$ cell responses as well as nNAbs and bNAbs and involving all antibody effector functions is likely of key importance for induction of robust protection. 


\section{Acknowledgements}

We are grateful to Takaji Wakita and Jens Bukh for JFH1 and J6CF isolates and HCV chimeras, respectively, and to Charles Rice for Huh-7.5 cells and the 9E10 antibody. We thank Steven Foung for generous provision of CBH-7, HC-1, HC-11, HC84-20, HC84-21, and HC84-22 monoclonal antibodies, and Arvind Patel for providing AP33, and Mansun Law for gift of AR3B. We are particularly thankful to Thomas Schulz and the members of his diagnostics team for help in the quantification of HCV core protein levels. We would also like to thank all members of the Institute for Experimental Virology for helpful suggestions and discussions.

Twincore is a joint venture of Hannover Medical School and the Helmholtz-Centre for infection research. T.P. was supported by a grant from the DZIF (HCV-vaccine), and T.P. and C.A.G. were supported by the Helmholtz-Alberta Initiative for Infectious Disease Research (HAI-IDR). 


\section{Materials and Methods}

\section{Plasmid constructs}

The renilla-luciferase harboring Jc1 [23] and Jc1- $\Delta$ HVR1 [10] viral cDNA clones with or without alanine substitutions within N-linked glycosylation-sites of E2 (N417A, N423A, N448A, N534A and N649A) were constructed by standard PCR-based techniques and verified by sequencing.

\section{HCVcc neutralization assay}

312 For inhibition of HCV infection, $200 \mu \mathrm{l}$ of a Huh7.5 cell suspension $\left(5 \times 10^{4}\right.$ cells per ml $)$ was seeded into 313 each well of a 96-well plate $24 \mathrm{~h}$ prior to inoculation. Luciferase reporter viruses were mixed with serial 314 dilutions of indicated serum/antibody concentrations and pre incubated for $1 \mathrm{~h}$. This mixture was used to 315 inoculate cells for $4 \mathrm{~h}$ in triplicates per dilution. Thereafter, $170 \mu \mathrm{l}$ of DMEM was added onto the cells. 316 Viral infection was determined 48 or $72 \mathrm{~h}$ after infection by removing the supernatant and lysing of the 317 cells by addition of passive lysis buffer or water and measurement of RLU using a 96-well plate reader 318 (Berthold).

\section{HCV pseudotyped viruses (HCVpp) neutralization assay}

$320 \mathrm{HCV}$ pseudotyped viruses (HCVpp) expressing a luciferase reporter were generated as described [35]. For 321 neutralization assays, Huh7.5 cells were plated on poly-lysine coated 96-well plates 1 day prior to 322 infection. HCVpp were diluted 1:10 and premixed with heat inactivated diluted sera (1:100) for $1 \mathrm{~h}$ at $32337^{\circ} \mathrm{C}$ followed by addition to Huh 7.5 cells. Six hours post-infection, the antibody-virus inoculum was 324 replaced with fresh culture medium. Cells were processed $48 \mathrm{~h}$ post-infection using the Bright-glo 325 luciferase assay system (Promega, Madison, WI, USA). Luminescence was measured using an Enspire 326 plate reader (Perkin-Elmer,Waltham, MA, USA). The neutralization activity was calculated using the 327 following formula: $\%$ neutralization $=($ pre-post $) /$ pre $\times 100$ where pre/post represent the luciferase activity 328 done after incubating with either the pre- or post-vaccination sera.

\section{Molecular modeling}

330 Modelling of the glycosylated core structure (PDB 4MWF) was performed using Glycoprotein Builder 331 (www.glycam.org). Glycans are depicted as wireframes; protein as a surface. The predicted CD81 binding 332 site and $\mathrm{mAb} \mathrm{HC} 1 / \mathrm{HC} 11$ epitopes (highlighted in green) were annotated using UCSF Chimera 333 (www.cgl.ucsf.edu/chimera) using a space fill model. Other amino acid side chains were coloured by 334 hydrophobicity (red = hydrophobic; blue = hydrophilic as determined by the Kyte-Doolittle scale). 


\section{References}

1 WHO. 2017. Hepatitis C. WHO fact sheet 164. WHO G, Switzerland:.

2 Tarr AW, Khera T, Hueging K, Sheldon J, Steinmann E, Pietschmann T, et al. Genetic diversity underlying the envelope glycoproteins of hepatitis $C$ virus: Structural and functional consequences and the implications for vaccine design. Viruses 2015;7:3995-4046.

3 Walker CM. Designing an HCV vaccine: a unique convergence of prevention and therapy? Curr Opin Virol 2017;23:113-9.

$4 \quad$ Houghton M. Prospects for prophylactic and therapeutic vaccines against the hepatitis $\mathrm{C}$ viruses. Immunol Rev 2011;239:99-108.

5 Fauvelle C, Colpitts CC, Keck ZY, Pierce BG, Foung SK, Baumert TF. Hepatitis C virus vaccine candidates inducing protective neutralizing antibodies. Expert Rev Vaccines 2016;15:1535-44.

6 Law JL, Chen C, Wong J, Hockman D, Santer DM, Frey SE, et al. A hepatitis C virus (HCV) vaccine comprising envelope glycoproteins gpE1/gpE2 derived from a single isolate elicits broad cross-genotype neutralizing antibodies in humans. PLoS One 2013;8:e59776.

7 Frey SE, Houghton M, Coates S, Abrignani S, Chien D, Rosa D, et al. Safety and immunogenicity of HCV E1E2 vaccine adjuvanted with MF59 administered to healthy adults. Vaccine 2010;28:6367-73.

8 Landi A, Law J, Hockman D, Logan M, Crawford K, Chen C, et al. Superior immunogenicity of HCV envelope glycoproteins when adjuvanted with cyclic-di-AMP, a STING activator or archaeosomes. Vaccine 2017;35:6949-56.

9 Cashman SB, Marsden BD, Dustin LB. The Humoral Immune Response to HCV: Understanding is Key to Vaccine Development. Front Immunol 2014;5:550.

10 Bankwitz D, Steinmann E, Bitzegeio J, Ciesek S, Friesland M, Herrmann E, et al. Hepatitis C virus hypervariable region 1 modulates receptor interactions, conceals the CD81 binding site, and protects conserved neutralizing epitopes. J Virol 2010;84:5751-63.

11 Prentoe J, Jensen TB, Meuleman P, Serre SB, Scheel TK, Leroux-Roels G, et al. Hypervariable region 1 differentially impacts viability of hepatitis $C$ virus strains of genotypes 1 to 6 and impairs virus neutralization. J Virol 2011;85:2224-34.

12 Zibert A, Schreier E, Roggendorf M. Antibodies in human sera specific to hypervariable region 1 of hepatitis C virus can block viral attachment. Virology 1995;208:653-61.

13 Weiner AJ, Geysen HM, Christopherson C, Hall JE, Mason TJ, Saracco G, et al. Evidence for immune selection of hepatitis $\mathrm{C}$ virus (HCV) putative envelope glycoprotein variants: potential role in chronic HCV infections. Proc Natl Acad Sci U S A 1992;89:3468-72.

14 Helle F, Goffard A, Morel V, Duverlie G, McKeating J, Keck ZY, et al. The neutralizing activity of anti-hepatitis $C$ virus antibodies is modulated by specific glycans on the E2 envelope protein. J Virol 2007;81:8101-11.

15 Helle F, Vieyres G, Elkrief L, Popescu Cl, Wychowski C, Descamps V, et al. Role of N-linked glycans in the functions of hepatitis $C$ virus envelope proteins incorporated into infectious virions. J Virol 2010;84:11905-15.

16 Goffard A, Callens N, Bartosch B, Wychowski C, Cosset FL, Montpellier C, et al. Role of N-linked glycans in the functions of hepatitis $C$ virus envelope glycoproteins. J Virol 2005;79:8400-9.

17 Kong L, Giang E, Nieusma T, Kadam RU, Cogburn KE, Hua Y, et al. Hepatitis C virus E2 envelope glycoprotein core structure. Science 2013;342:1090-4.

18 Khan AG, Whidby J, Miller MT, Scarborough H, Zatorski AV, Cygan A, et al. Structure of the core ectodomain of the hepatitis $C$ virus envelope glycoprotein 2. Nature 2014;509:381-4.

19 Keck ZY, Li TK, Xia J, Gal-Tanamy M, Olson O, Li SH, et al. Definition of a conserved immunodominant domain on hepatitis C virus E2 glycoprotein by neutralizing human monoclonal antibodies. J Virol 2008;82:6061-6. 
20 Pierce BG, Keck ZY, Lau P, Fauvelle C, Gowthaman R, Baumert TF, et al. Global mapping of antibody recognition of the hepatitis $C$ virus E2 glycoprotein: Implications for vaccine design. Proc Natl Acad Sci U S A 2016.

21 Keck ZY, Xia J, Wang Y, Wang W, Krey T, Prentoe J, et al. Human monoclonal antibodies to a novel cluster of conformational epitopes on HCV E2 with resistance to neutralization escape in a genotype 2a isolate. PLoS Pathog 2012;8:e1002653.

22 Keck ZY, Saha A, Xia J, Wang Y, Lau P, Krey T, et al. Mapping a region of hepatitis C virus E2 that is responsible for escape from neutralizing antibodies and a core CD81-binding region that does not tolerate neutralization escape mutations. J Virol 2011;85:10451-63.

23 Pietschmann T, Kaul A, Koutsoudakis G, Shavinskaya A, Kallis S, Steinmann E, et al. Construction and characterization of infectious intragenotypic and intergenotypic hepatitis $C$ virus chimeras. Proc Natl Acad Sci U S A 2006;103:7408-13.

24 Reiss S, Rebhan I, Backes P, Romero-Brey I, Erfle H, Matula P, et al. Recruitment and activation of a lipid kinase by hepatitis $C$ virus NS5A is essential for integrity of the membranous replication compartment. Cell Host Microbe 2011;9:32-45.

25 Bitzegeio J, Bankwitz D, Hueging K, Haid S, Brohm C, Zeisel MB, et al. Adaptation of hepatitis C virus to mouse CD81 permits infection of mouse cells in the absence of human entry factors. PLoS Pathog 2010;6:e1000978.

26 Whidby J, Mateu G, Scarborough H, Demeler B, Grakoui A, Marcotrigiano J. Blocking hepatitis C virus infection with recombinant form of envelope protein 2 ectodomain. J Virol 2009;83:11078-89.

27 Kachko A, Frey SE, Sirota L, Ray R, Wells F, Zubkova I, et al. Antibodies to an interfering epitope in hepatitis $C$ virus $E 2$ can mask vaccine-induced neutralizing activity. Hepatology 2015;62:1670-82.

28 Zhang $\mathrm{P}$, Zhong L, Struble EB, Watanabe $\mathrm{H}$, Kachko A, Mihalik K, et al. Depletion of interfering antibodies in chronic hepatitis $C$ patients and vaccinated chimpanzees reveals broad cross-genotype neutralizing activity. Proc Natl Acad Sci U S A 2009;106:7537-41.

29 Akazawa D, Moriyama M, Yokokawa H, Omi N, Watanabe N, Date T, et al. Neutralizing antibodies induced by cell culture-derived hepatitis $C$ virus protect against infection in mice. Gastroenterology 2013;145:447-55 e1-4.

30 Yokokawa H, Higashino A, Suzuki S, Moriyama M, Nakamura N, Suzuki T, et al. Induction of humoural and cellular immunity by immunisation with HCV particle vaccine in a non-human primate model. Gut 2016.

31 Stamataki Z, Coates S, Evans MJ, Wininger M, Crawford K, Dong C, et al. Hepatitis C virus envelope glycoprotein immunization of rodents elicits cross-reactive neutralizing antibodies. Vaccine 2007; 25:7773-84.

32 Vietheer PT, Boo I, Gu J, McCaffrey K, Edwards S, Owczarek C, et al. The core domain of hepatitis $C$ virus glycoprotein E2 generates potent cross-neutralizing antibodies in guinea pigs. Hepatology 2017;65:1117-31.

33 Kong L, Lee DE, Kadam RU, Liu T, Giang E, Nieusma T, et al. Structural flexibility at a major conserved antibody target on hepatitis C virus E2 antigen. Proc Natl Acad Sci U S A 2016.

34 Horwitz JA, Bar-On Y, Lu CL, Fera D, Lockhart AAK, Lorenzi JCC, et al. Non-neutralizing Antibodies Alter the Course of HIV-1 Infection In Vivo. Cell 2017;170:637-48 e10.

35 Hsu M, Zhang J, Flint M, Logvinoff C, Cheng-Mayer C, Rice CM, et al. Hepatitis C virus glycoproteins mediate $\mathrm{pH}$-dependent cell entry of pseudotyped retroviral particles. Proc Natl Acad Sci U S A 2003;100:7271-6.

36 Owsianka AM, Timms JM, Tarr AW, Brown RJ, Hickling TP, Szwejk A, et al. Identification of conserved residues in the E2 envelope glycoprotein of the hepatitis C virus that are critical for CD81 binding. J Virol 2006;80:8695-704. 
2

3

442937 Drummer HE, Boo I, Maerz AL, Poumbourios P. A conserved Gly436-Trp-Leu-Ala-Gly-Leu-Phe-Tyr 5430 motif in hepatitis C virus glycoprotein E2 is a determinant of CD81 binding and viral entry. J Virol $7431 \quad 2006 ; 80: 7844-53$.
13

14

15

16

17

18

19

20

21

22

23

24

25

26

27

28

29

30

31

32

33

34

35

36

37

38

39

40

41

42

43

44

45

46

47

48

49

50

51

52

53

54

55

56

57

58

59

60

61

62

63

64

65 


\section{Figure Legends}

Figure 1: Schematic and structure of HCV E2 and characterization of mutant viruses. (A) N-linked glycosylation sites (NGS) in the HCV E2 ectodomain are indicated by inverted open triangles. For positional referencing, a graphic chart of the E2 protein is located directly below, with two hypervariable regions and the intergenic variable region (HVR1, HVR2 and igVR) highlighted, and the stem region of E2 marked in light grey. All numbering is relative to the full-length ORF position in the H77 (GT1a) reference strain (accession number NC_004102) while numbers in brackets indicate the homologous position in the J6 (GT2a) E2 ectodomain. The HVR1 region and glycan sites, which were deleted, are indicated in red. Zoomed in region highlights the viral CD81 binding site with key contact residues displayed in blue [36, 37]. Amino acid important for binding of bNAbs HC1, HC11, and HC84-like antibodies are highlighted with colored inverted triangles (pink, $\mathrm{HC1}$; green $\mathrm{HC} 11$, orange HC84-series). ** indicates indels responsible for length differences between H77 (GT1a) and J6 E2 (GT2a). Note that HC84.20 also binds to Y613 and W616 and HC84.22 also binds to W616. (B) Structural modelling of the E2 core domain with the $\mathrm{CD} 81, \mathrm{HC} 1$ and $\mathrm{HC} 11$ binding sites highlighted in green. Glycosylation was modelled using Glycoprotein Builder and are shown and numbered according to the H77 (GT1a) reference strain. The E2 protein is colored by hydrophobicity of aa side chains (red and blue represent hydrophobic and hydrophilic residues, respectively) and green shows antibody or CD81 contact residues. (C) Replication of mutant viruses with alanine substitutions at indicated positions with or without deletion of the HVR1 within the E2 protein. Viral replication was determined by renilla-luciferase activity of cell lysates $48 \mathrm{~h}$ after transfection ( $\mathrm{n}=3$, duplicates each; SD). (D) Specific infectivity of particles. Released virus was normalized to equal amounts of core and was used to infect Huh-7.5 cells. Infection is displayed as RLU per well ( $n=3$, measured in triplicates each; SD). The dotted line depicts the background of the assay as determined by measurements of uninfected cell lysates.

Figure 2: Precipitation of mutant viruses with monoclonal antibodies and with GST-CD81-LEL. Mutant viruses were normalized to equal amounts of core and incubated with either HC-1 (A), HC-11 (B) or with GST-C81-LEL (C), respectively. Virus-antibody or virus-CD81-LEL-GST complexes were precipitated by addition of protein $\mathrm{G}$ or glutathione coated beads. Viral core protein was determined to measure bound viral particles. Data are expressed as fold over control (antibody RO4 or GST) and compared to WT virus ( $\mathrm{n}=4$, ANOVA, Friedman, Dunn's multiple comparison, $\mathrm{p}<0.05(*), \mathrm{p}<0.01(* *)$, $\mathrm{p}<0.001(* * *))$. Symbols represent individual IPs. 
Figure 3: Neutralization of Jc1 and mutant viruses by antibodies or by receptor competition with GST-CD81LEL. Viruses were incubated with serial dilutions of given antibodies (A) or GST-CD81-LEL (B) and infection efficiency was quantified by luciferase assays. Selected dose-response curves are plotted in panel (C). Radar plots in (A) highlight $\mathrm{IC}_{90}$ values $(\mu \mathrm{g} / \mathrm{ml})$ for antibody neutralization, and $\mathrm{IC}_{90}$ values of GST-CD81-LEL receptor competition in panel (B), respectively. The $\mathrm{IC}_{90}$ values were calculated using non-linear regression method on graph pad version 6. Abbreviations: RLU, Relative light units.

Figure 4: Neutralization of viruses by GT1 and GT2 patient sera. Indicated viruses were mixed with serial dilutions of sera of uninfected (A, \#1-3), GT1 (B, \#1-3) or GT2 infected (C, \#1-3) individuals. Infection efficiency was quantified by inoculation of Huh-7.5 cells and subsequent luciferase assays. It is plotted relative to control infections in the absence of human serum. Means \pm SD ( $n=2(A / B / C \# 1-2)$ or $3(\mathrm{~A} / \mathrm{B} / \mathrm{C} \# 3))$.

Figure 5: Cross-binding of vaccine-induced antibodies to HCV GT1 to 6 glycoproteins. (A) Recombinant E2 proteins $(2 \mu \mathrm{g} / \mathrm{ml})$ from different HCV GTs were immobilized on ELISA plates. (B) Alternatively, E1-E2 heterodimers prepared from virus transfected cells were used to determine antibody cross binding. Plates were incubated with serial dilutions of mouse sera from vaccinated and mock treated animals, and bound antibodies were detected with a secondary anti-mouse antibody coupled to horseradish peroxidase (HRP). The background detected upon incubation with sera from mock vaccinated animals was subtracted and the end point binding titers were calculated based on the median effect method as described in the materials and methods section. Raw data are provided in the supplementary figure $2 \mathrm{~A}$ and B. (C) Antibody cross-binding expressed relative to vaccination with the parental J6-E2 protein and measured by ELISAs using recombinant E2 (left panel) or E1-E2 heterodimers extracted from cells with infectious HCV (right panel). Horizontal bars represent the median value of cross binding relative to the wildtype J6-E2 vaccination.

Figure 6: Quantification of vaccine-induced neutralizing antibody responses. (A) Neutralization of autologous Jc1 and of heterologous GT5A reporter virus by vaccine induced mouse antibodies and human antibodies from HCV patients. Viruses were incubated with given doses of antibodies and infection efficiency was determined and expressed relative to infections conducted in the absence of antibodies. Means \pm SD of three technical replicates are shown. (B) Influence of vaccine-induced mouse IgG on neutralization by human neutralizing antibodies. Purified mouse IgGs $(100 \mu \mathrm{g} / \mathrm{ml}$; pooled from all animals in one group) were mixed with human $\mathrm{IgG}(10 \mu \mathrm{g} / \mathrm{ml})$ from an $\mathrm{HCV}$ patient, pre-incubated with GT5a reporter virus for $1 \mathrm{~h}$, and then used to inoculate Huh-7.5 cells. Infection efficiency was determined as above and is expressed relative to infections conducted in the absence of any IgGs. Means \pm SD ( $n=3$ ). 
Figure 7: Vaccination with E1E2 heterodimers with or without glycosylation site mutation induces comparable neutralizing antibody responses. (A) Effect of $\mathrm{N}$-glycosylation mutations on E1E2 interaction with CD81-LEL. Purified WT or N-glycosylation mutant (N417Q and N532Q) E1E2 proteins (25, 50 and $100 \mathrm{ng}$ ) were added to CD81-LEL coated wells or BSA coated wells (single $100 \mathrm{ng}$ E1E2 dose) as a negative control. Bound E1E2 antigens were detected by anti-E2 (H53) mAb. Values are shown as a percentage of WT E1E2 binding at the highest dose tested $\left.(100 \mathrm{ng}) .{ }^{*}\right)$ Designates $\mathrm{p}<0.05$ respective to WT (100 ng) by One way ANOVA; Tukey post-hoc test. (B) HCV E2 binding antibodies induced by vaccination. WT recombinant E2 (384-661) H77c antigens were coated to ELISA plates in triplicate and probed with post-vaccinated mice sera. Binding of E2-specific antibodies from WT, N417Q, and N532Q (1000, 2000, 4000-fold dilutions) vaccinated animals compared to control (C) sera (1000-fold dilution) were detected by anti-mouse HRP conjugated secondary antibody and peroxidase substrate. The OD450$570 \mathrm{~nm}$ values (mean and SEM) plotted vs serum dilution. Shown is one representative of three independent experiments. (C) Competition of mice antisera with HCV cross-neutralizing monoclonal antibodies (mAb) to E1E2. Microtiter wells containing GNA-purified E1E2 H77c were incubated with diluted terminal antiserum (1:100) from control (C) or E1E2 (WT, N417Q or N532Q antigen) vaccinated mice. After washing, plates were incubated with anti-HCV mAbs. Bound AR3B, 1:7 and HC33.4 were detected with anti-human alkaline phosphatase-conjugated secondary antibody and biotin-AP33 detected using neutravidin-alkaline phosphatase. The percentages of mAb binding were calculated relative to the amount of $\mathrm{mAb}$ bound in the absence of antiserum. Shown are mean values for each group \pm range from three independent experiments. $\left(^{*}\right)$ designates $\mathrm{p}<0.05$ respective to the control group by One way ANOVA; Tukey post-hoc test. (D) Comparison of the HCVpp (H77) neutralization response from WT and N-glycosylation mutant E1E2 vaccinated mice. Neutralization assay using homologous HCVpp H77 (1a) were performed using pre- and post-vaccinated sera (1:100) and the group means with SEMs plotted from representatives of three independent experiments. Positive control: Anti-CD81 mAb [1 $\mu \mathrm{g} / \mathrm{ml}]$ ). Abbreviations: OD, optical density. 
Figure 1

A

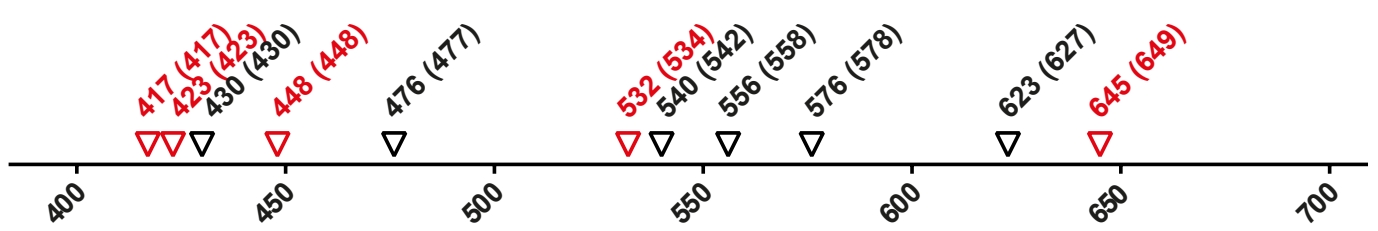

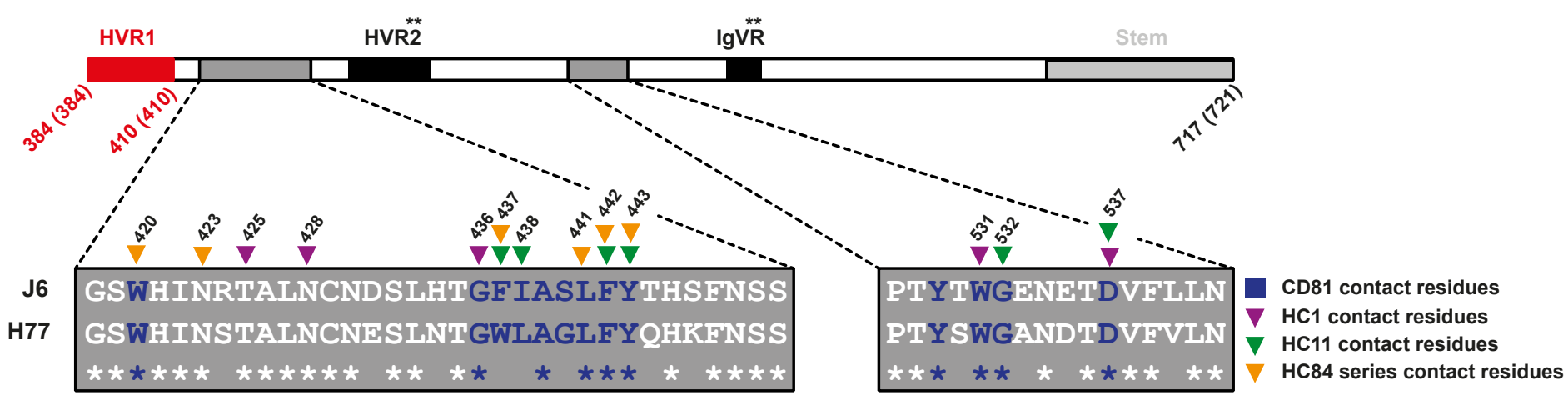

Consensus GSWH INRTALNCNDSLNTGF I AGLFYTHKFNSS Conservation

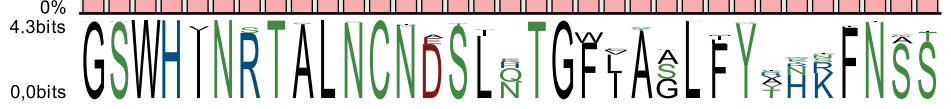

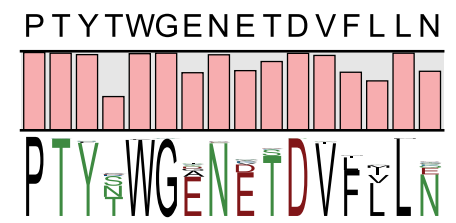

Conservation over all genotypes

B

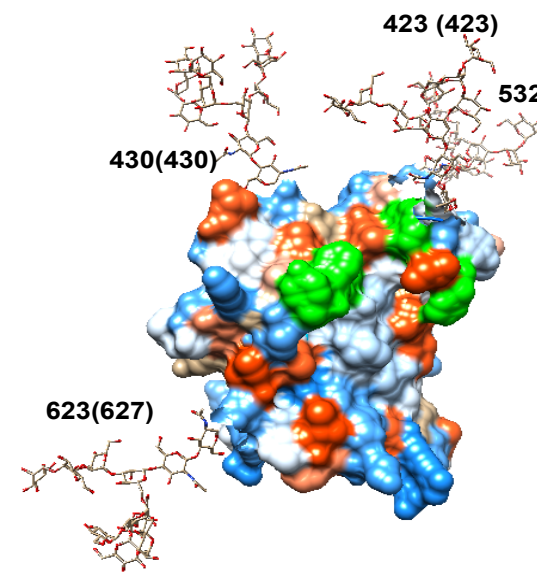

CD81 contact residue

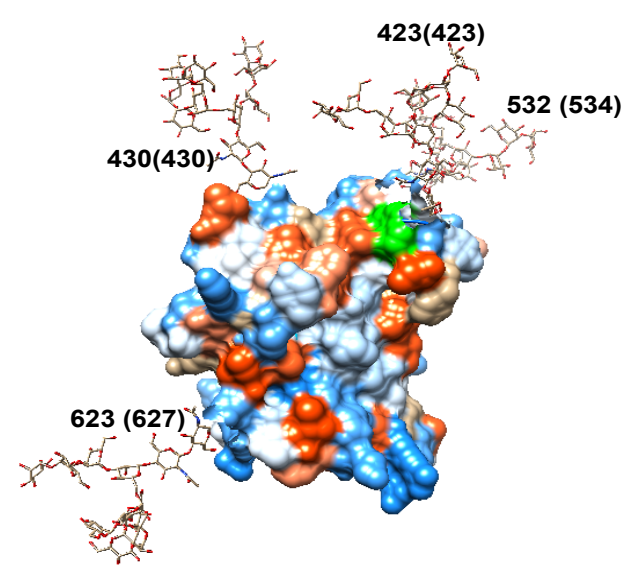

HC1 contact residue

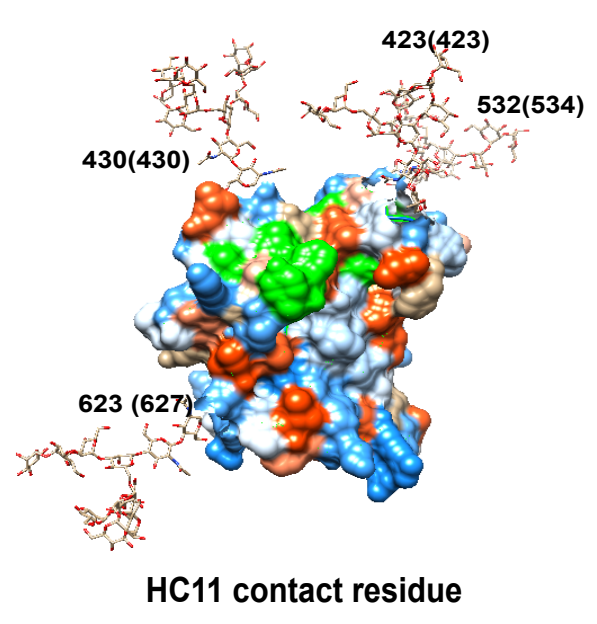

C

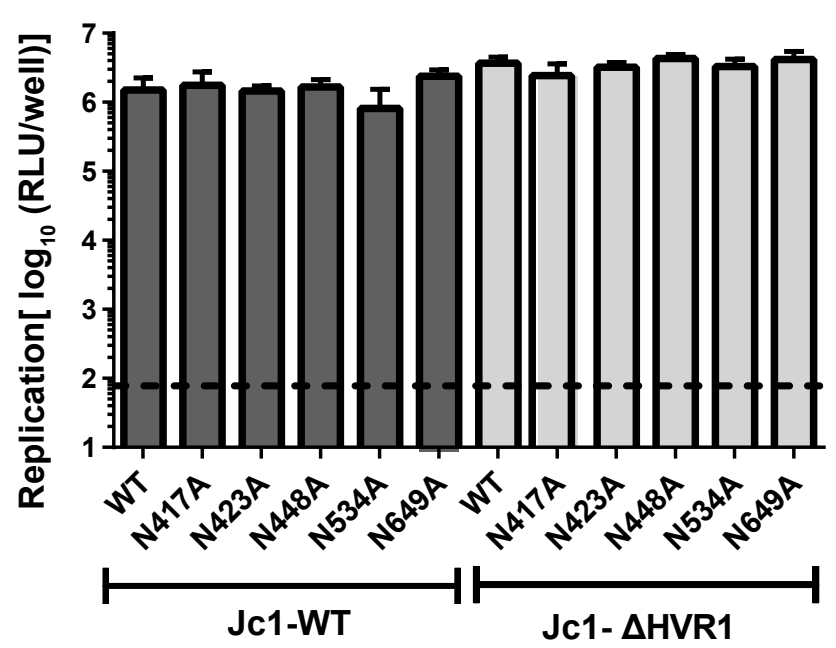

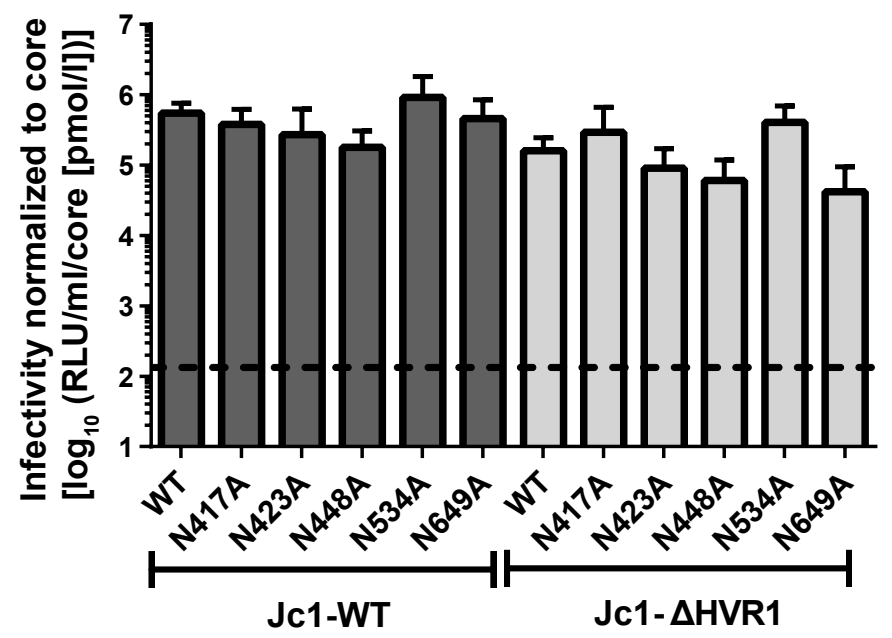


Figure 2

A

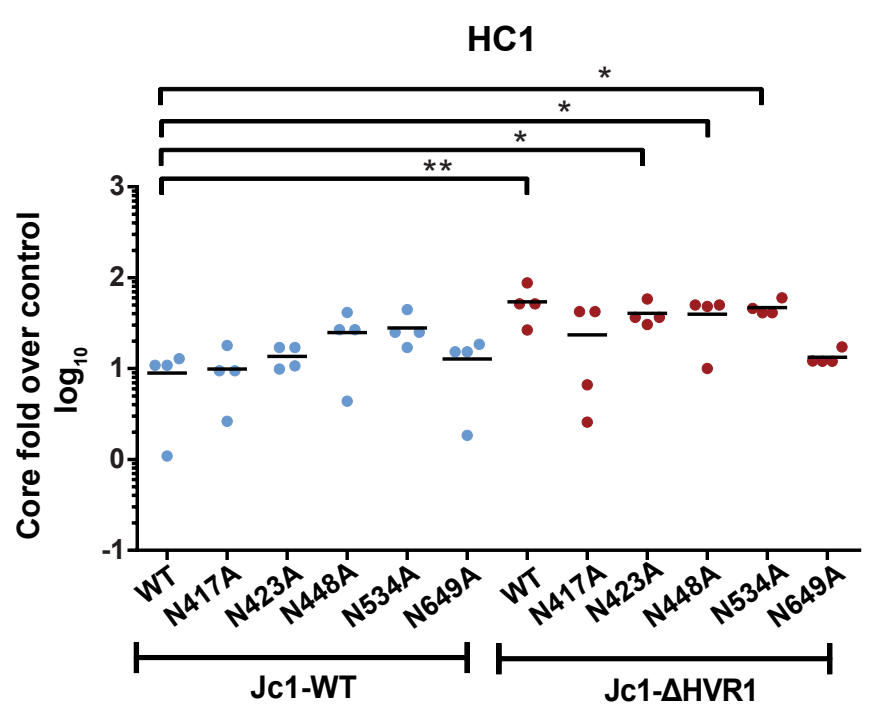

B

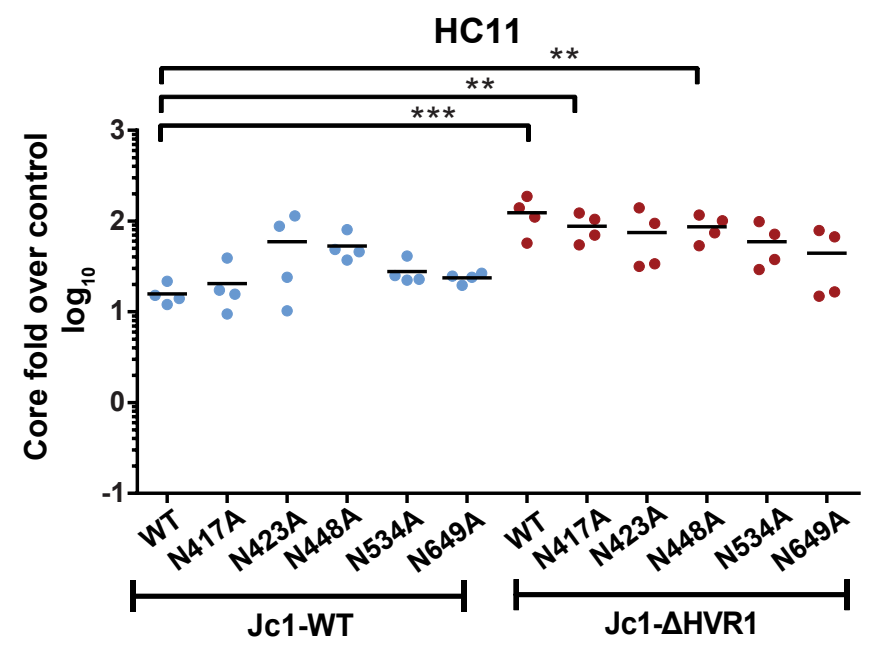

C

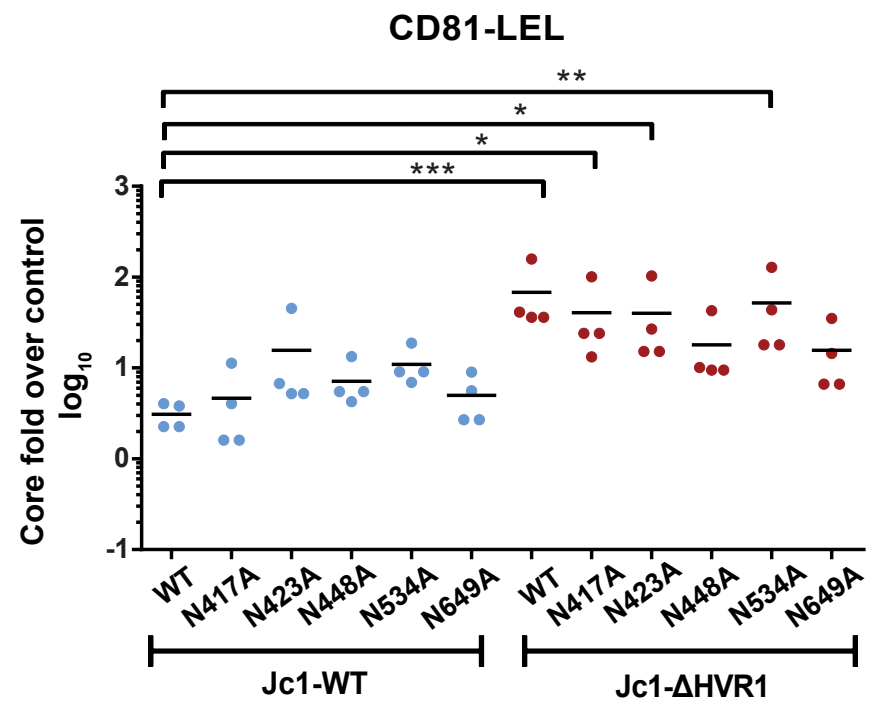




\section{Figure 3}

\section{A Jc1-WT}
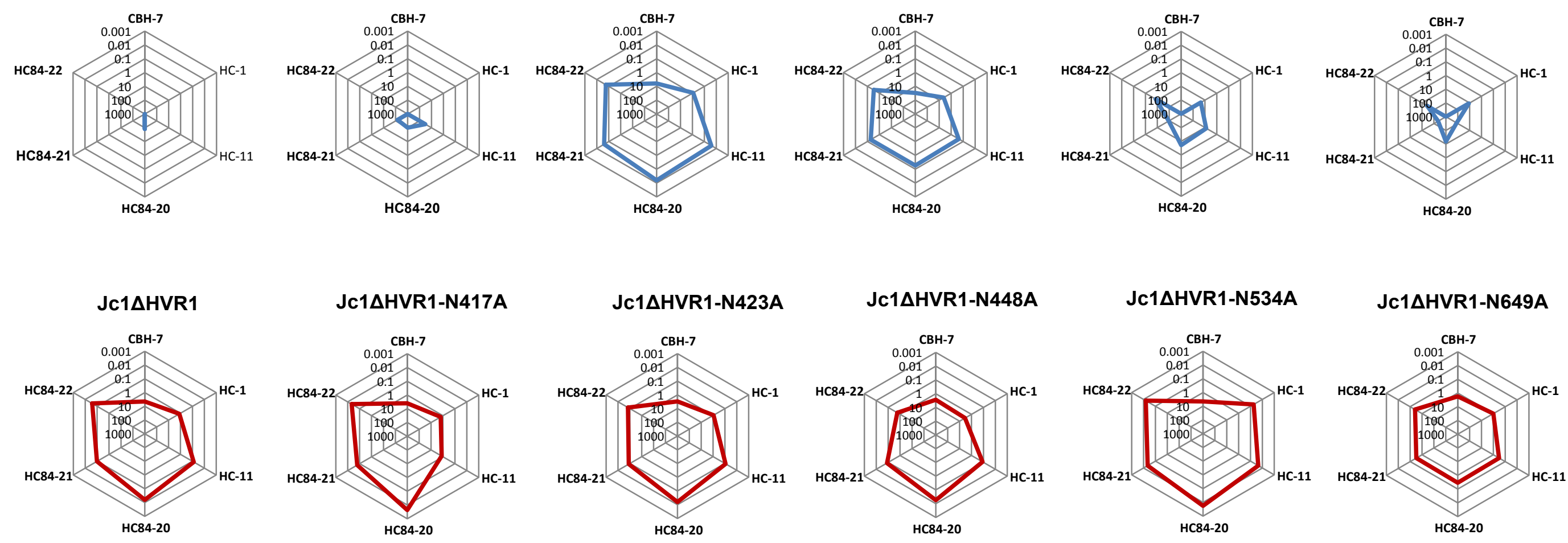

B

\section{CD81-LEL}
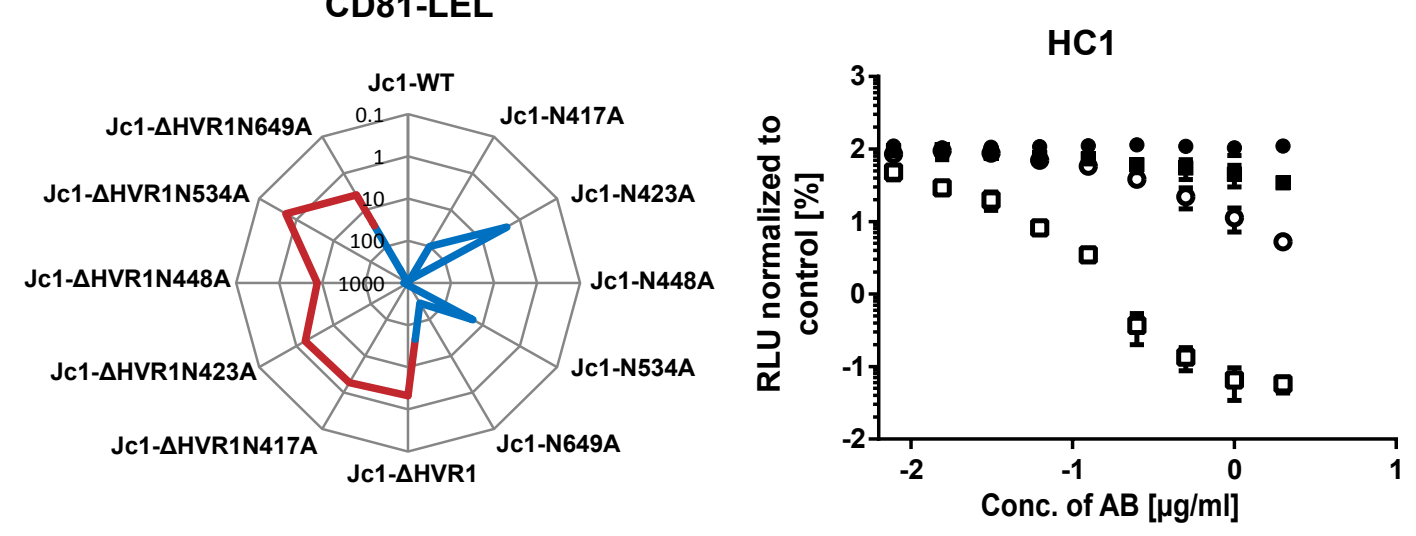

HC11

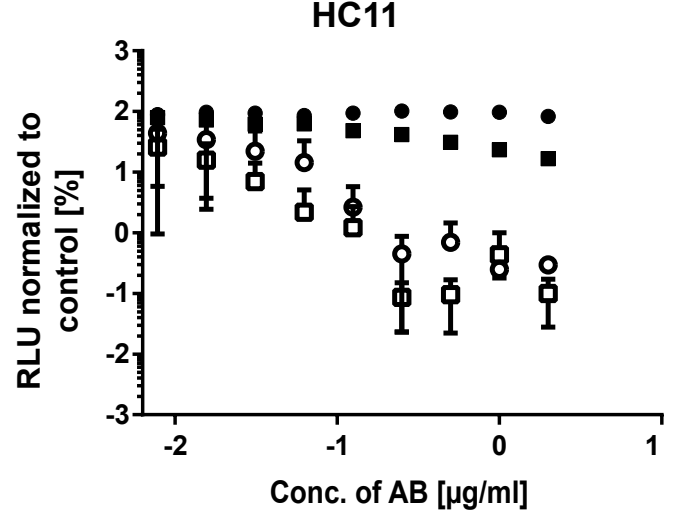

CD81-LEL

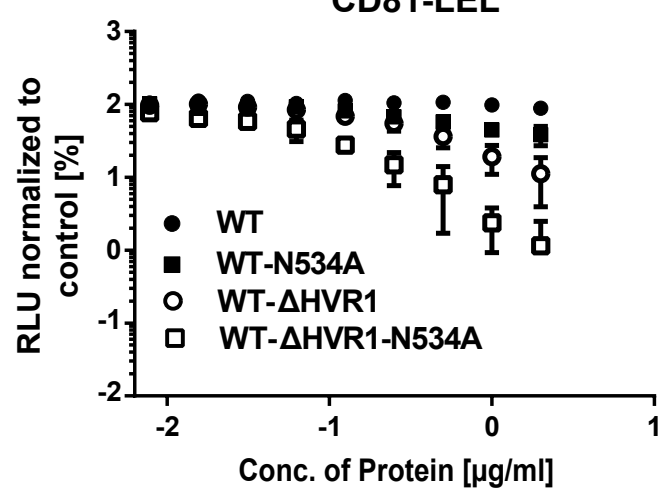


A
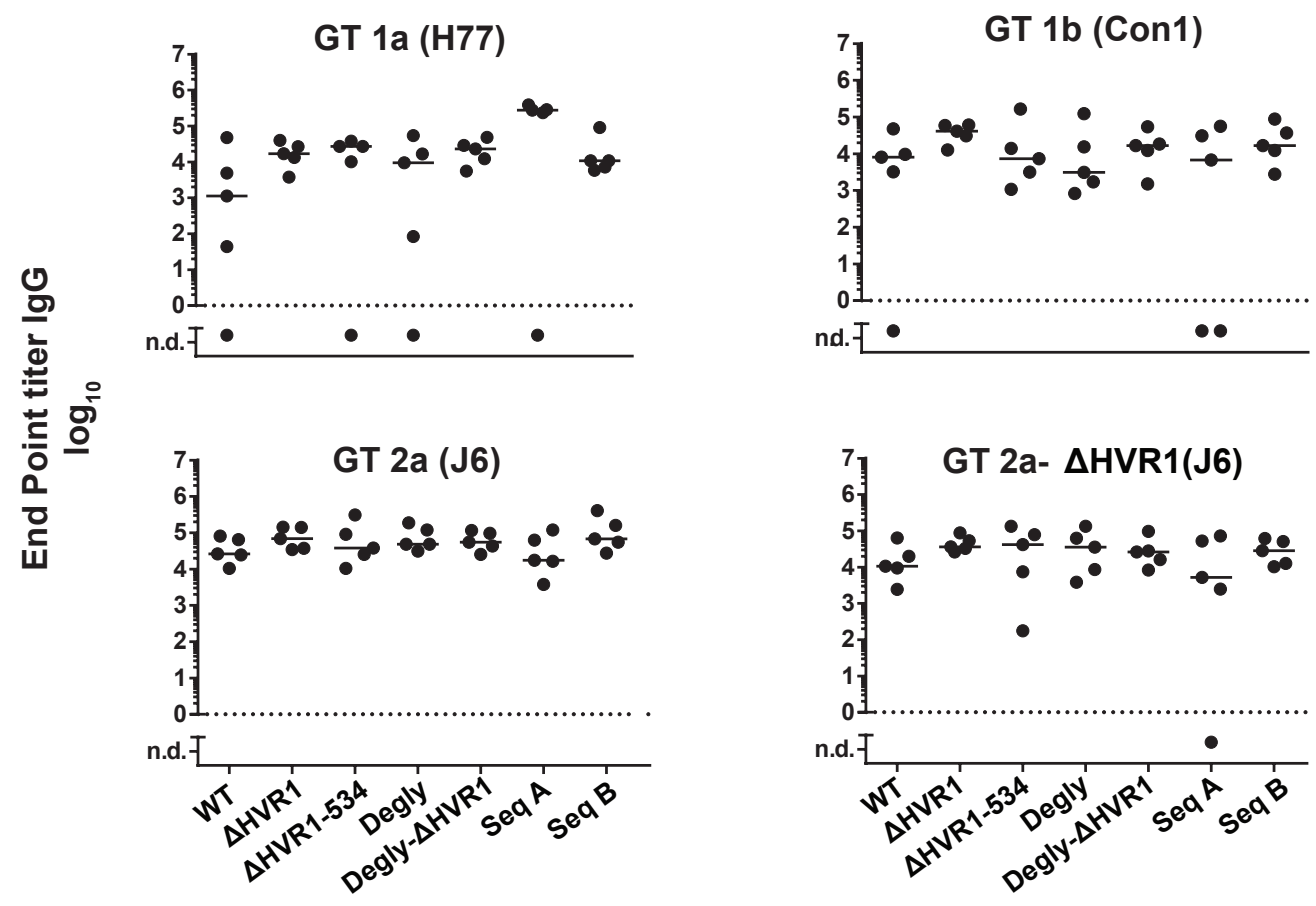

B
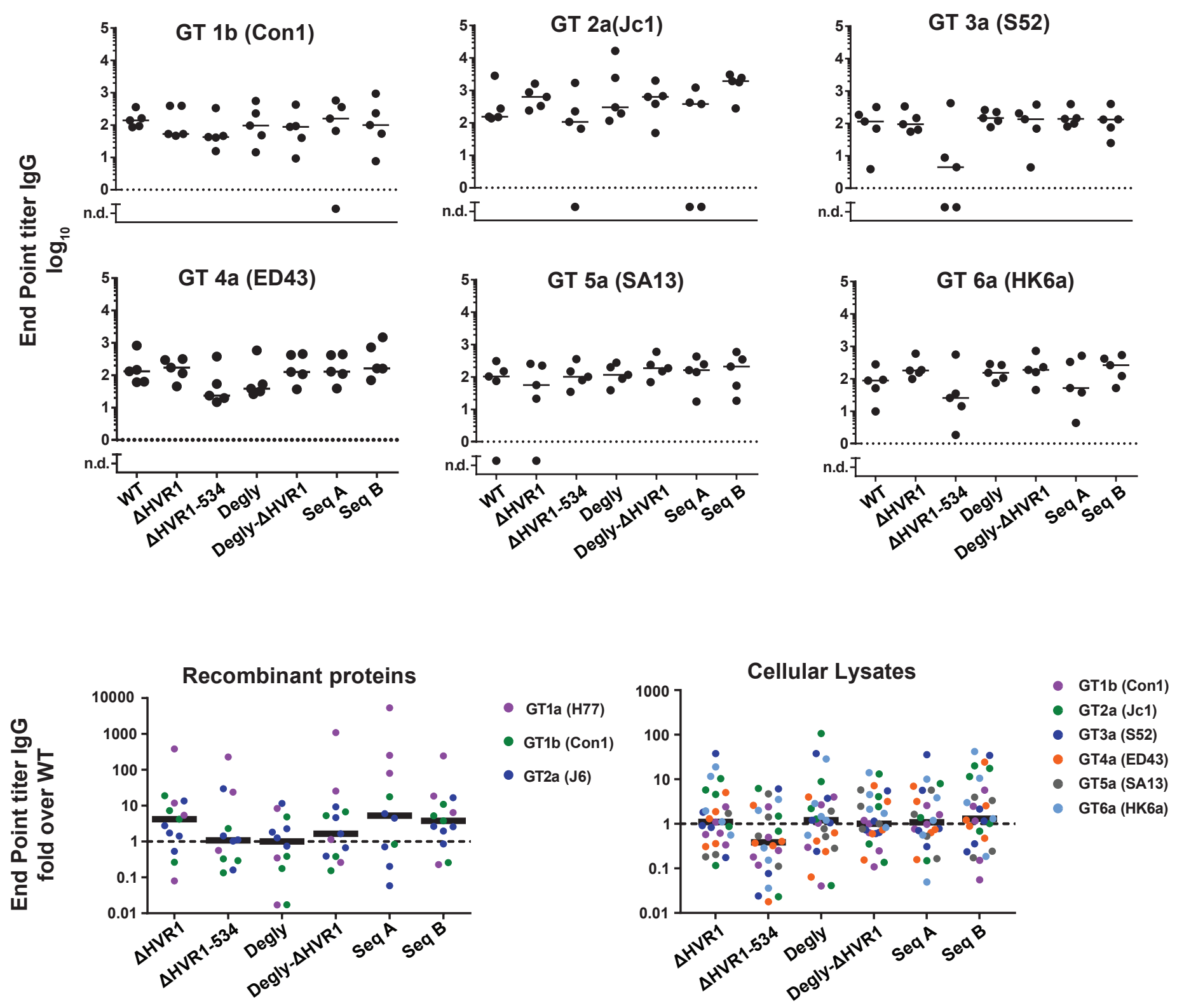
Figure 6
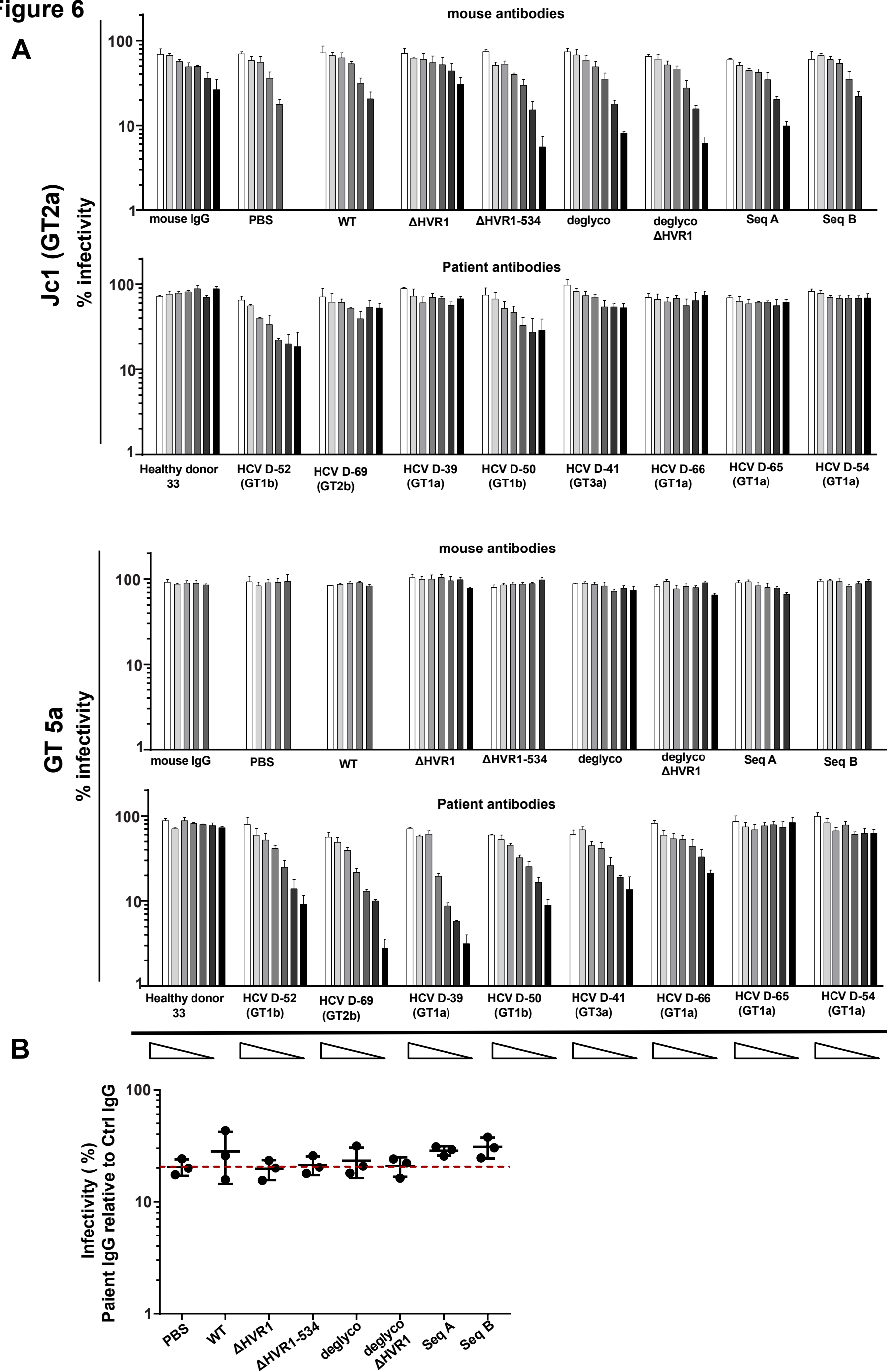

口 $7.9 \mu \mathrm{g} / \mathrm{ml}$

$15.6 \mu \mathrm{g} / \mathrm{ml}$

口 $31.2 \mu \mathrm{g} / \mathrm{ml}$

口 $62.5 \mu \mathrm{g} / \mathrm{m}$

$125 \mu \mathrm{g} / \mathrm{ml}$

$250 \mu \mathrm{g} / \mathrm{ml}$

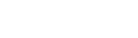


A

CD81LEL
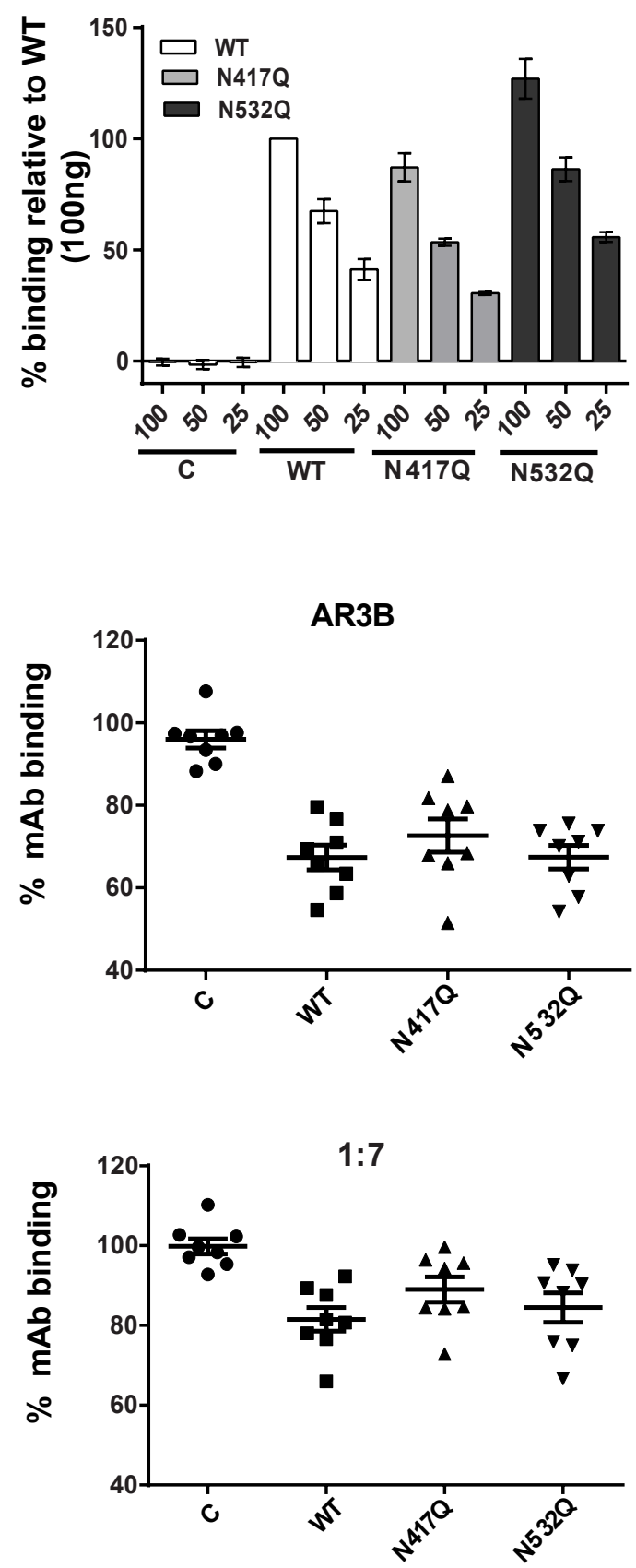

D

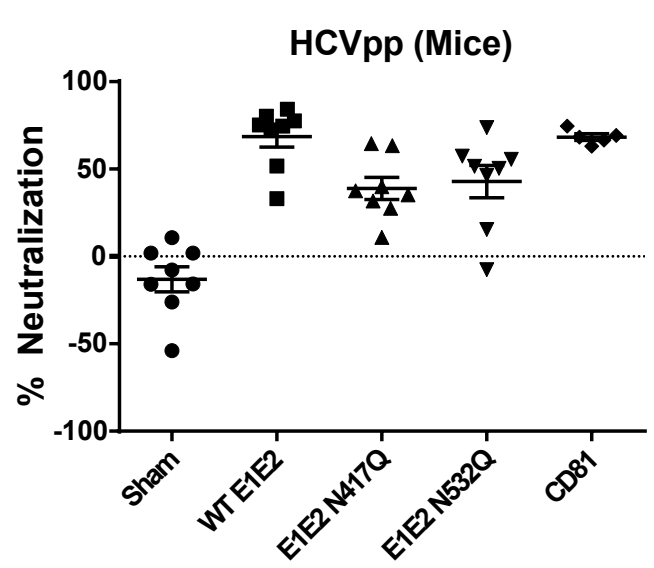

B

rE2-WT H77 ELISA
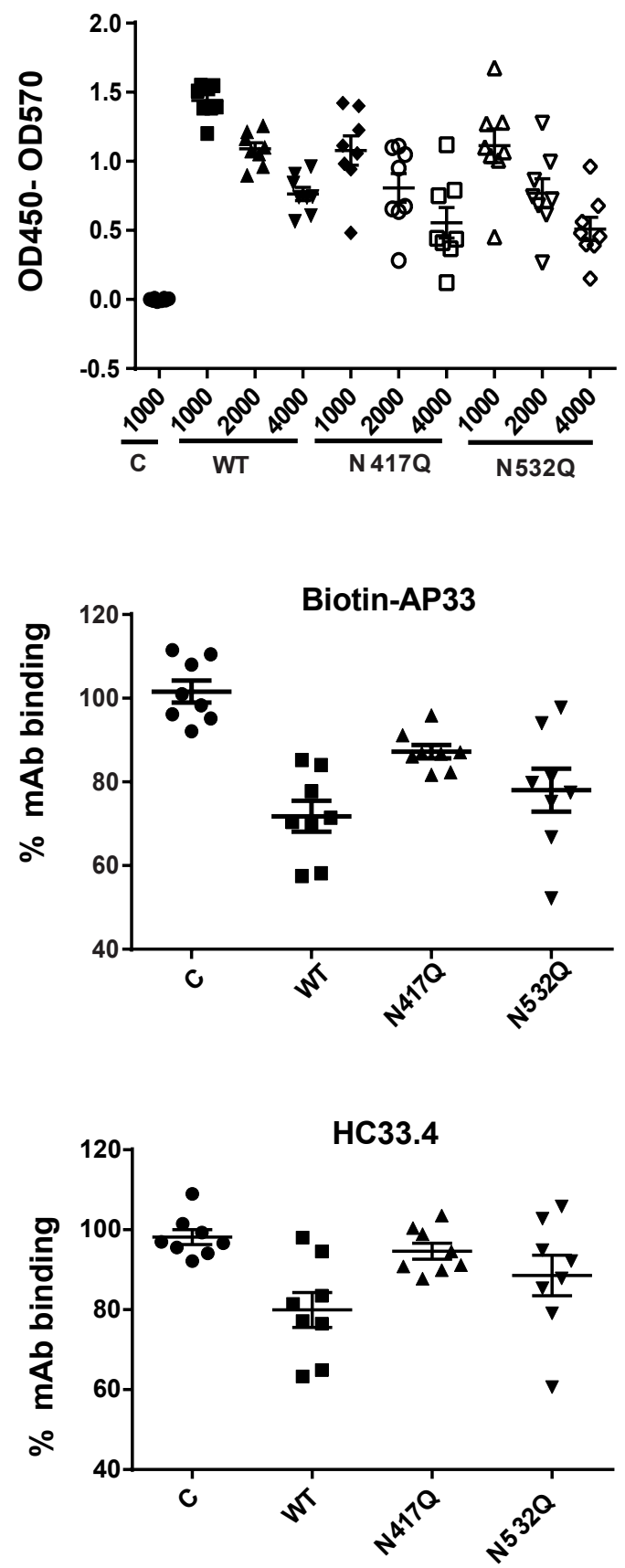


\section{Parental HCV}

Conserved epitopes/CD81 binding site partially occluded

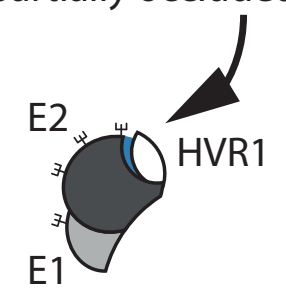

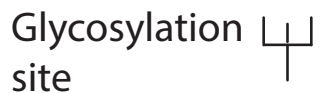

Conserved epitopes/ CD81 binding site

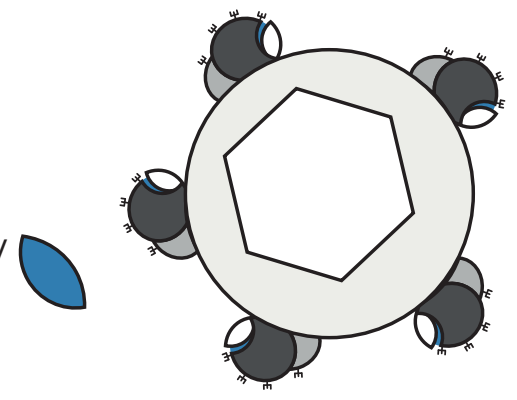

\section{Recombinant E2}

HVR1-deleted

deglycosylated

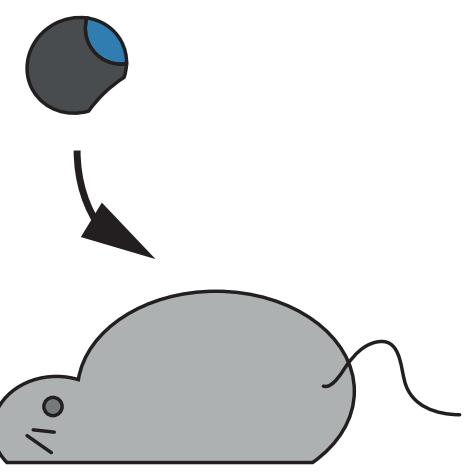

\section{Mutant HCV}

Conserved epitopes/CD81 binding site more exposed

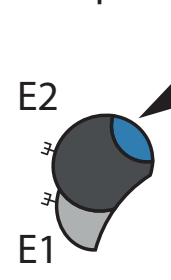<smiles>C1CCCC1</smiles>

HVR1-deleted

de-glycosylated

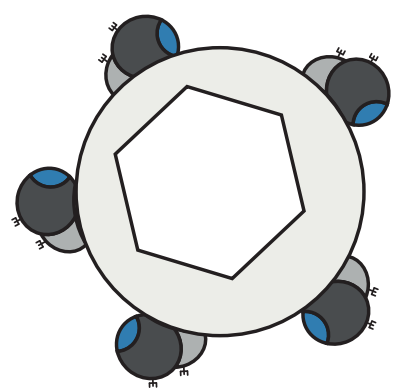

\section{Antibody response}

Strong binding to recombinant protein Enhanced cross-binding to E2 Comparable binding to E1-E2 Strain-specific neutralization No cross-neutralization 


\section{Highlights}

- High resolution mapping of the impact of HVR1 and glycosylation sites on CD81 binding, antibody binding and virus neutralization

- Viral mutants lacking HVR1 and selected glycosylation sites are functional and they expose the viral CD81 binding site and conserved cross-neutralization epitopes

- E2 proteins with these mutations induce cross-binding and non-interfering antibodies in mice 